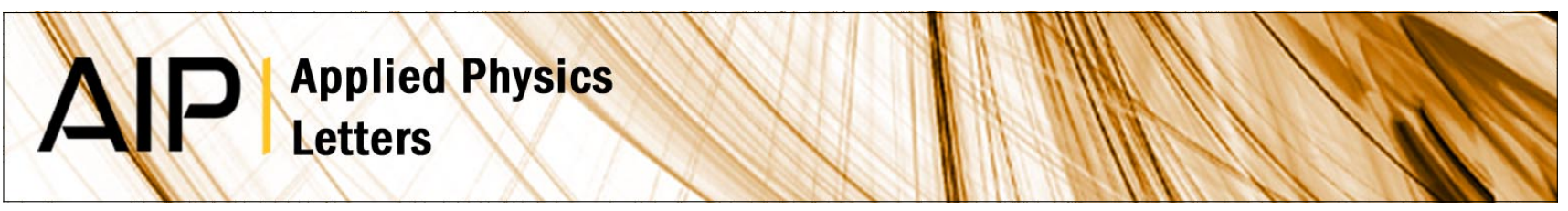

\title{
Enhanced sheath heating in capacitively coupled discharges due to non- sinusoidal voltage waveforms
}

T. Lafleur, R. W. Boswell, and J. P. Booth

Citation: Appl. Phys. Lett. 100, 194101 (2012); doi: 10.1063/1.4712128

View online: http://dx.doi.org/10.1063/1.4712128

View Table of Contents: http://apl.aip.org/resource/1/APPLAB/v100/i19

Published by the American Institute of Physics.

\section{Related Articles}

Toroidal ripple transport of beam ions in the mega-ampère spherical tokamak

Phys. Plasmas 19, 072514 (2012)

Global model of a gridded-ion thruster powered by a radiofrequency inductive coil

Phys. Plasmas 19, 073512 (2012)

Optics of ion beams for the neutral beam injection system on HL-2A Tokamak

Rev. Sci. Instrum. 83, 073307 (2012)

Initial measurements of fast ion loss in KSTAR

Rev. Sci. Instrum. 83, 10D305 (2012)

Thermo-magneto coupling in a dipole plasma

Phys. Plasmas 19, 072303 (2012)

\section{Additional information on Appl. Phys. Lett.}

Journal Homepage: http://apl.aip.org/

Journal Information: http://apl.aip.org/about/about_the_journal

Top downloads: http://apl.aip.org/features/most_downloaded

Information for Authors: http://apl.aip.org/authors

\section{ADVERTISEMENT}

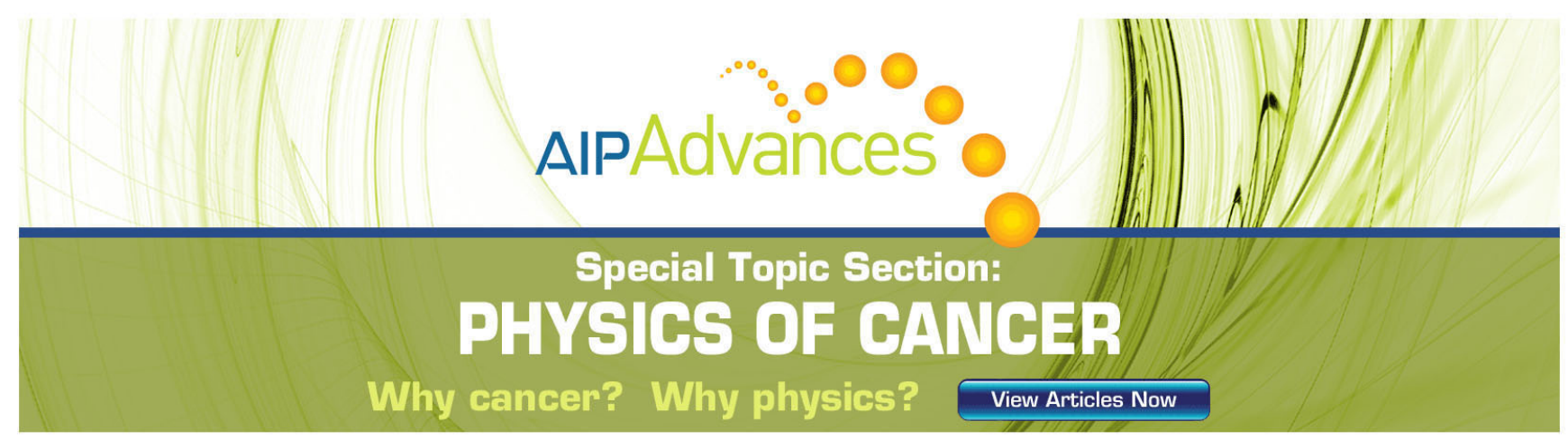




\title{
Enhanced sheath heating in capacitively coupled discharges due to non-sinusoidal voltage waveforms
}

\author{
T. Lafleur, ${ }^{1, a)}$ R. W. Boswell, ${ }^{2}$ and J. P. Booth ${ }^{3}$ \\ ${ }^{1}$ Johannesburg, South Africa \\ ${ }^{2}$ Space Plasma, Power and Propulsion Group, Research School of Physics and Engineering, The Australian \\ National University, Canberra ACT 0200, Australia \\ ${ }^{3}$ LPP-CNRS, Ecole Polytechnique, 91128 Palaiseau, France
}

(Received 2 March 2012; accepted 23 April 2012; published online 7 May 2012)

\begin{abstract}
Through the use of particle-in-cell simulations, we demonstrate that the power deposition in capacitively coupled discharges (in argon) can be increased by replacing sinusoidal waveforms with Gaussian-shaped voltage pulses (with a repetition frequency of $13.56 \mathrm{MHz}$ ). By changing the Gaussian pulse width, electron heating can be directly controlled, allowing for an increased plasma density and ion flux for the same gas pressure and geometrical operating conditions. Analysis of the power deposition profiles and electron distribution functions shows that enhanced electron-sheath heating is responsible for the increased power absorption. (c) 2012 American Institute of Physics. [http://dx.doi.org/10.1063/1.4712128]
\end{abstract}

An important factor in many material processing applications is the separate control over the ion flux and ion energy in capacitively coupled plasma (CCP) discharges; factors which affect such properties as the deposition and etching rates. ${ }^{1}$ In conventional single-frequency sinusoidally excited discharges, the ion flux and ion energy are coupled, allowing for limited control over each without changing the other. This has subsequently led to research and development with non-harmonic dual or multi frequency systems, where improved control over each factor can be obtained. ${ }^{1}$ It has recently been proposed, ${ }^{2,3}$ and experimentally ${ }^{4}$ and numerically $^{3,5}$ verified, that by making use of waveforms consisting of multiple harmonics, it is possible to develop an electrical asymmetry effect (EAE) in an otherwise geometrically symmetric plasma system. This has allowed the bias voltage (and hence ion energy) to be controlled by adjusting the voltage waveform, while keeping the plasma density and ion flux essentially constant, thus adding an additional level of control. The majority of work in this area has so far focussed on the use of a fundamental frequency, with an even harmonic. ${ }^{5-8}$ By changing the phase difference between the two signals, significant control over the bias voltage and ion energy has been demonstrated. ${ }^{5}$

The discovery of the EAE effect has led to a number of further investigations, both numerically ${ }^{5,6,8}$ and experimentally, ${ }^{9}$ on the fundamental physics, and practical use of these non-sinusoidal voltage waveforms. For example, numerical studies ${ }^{7}$ have demonstrated that it is possible to use the EAE to modify a geometrically asymmetrical system, and force it to become symmetrical. Other experimental work, such as that in Ref. 9, has used Gaussian-type pulses to control the growth of amorphous and nanocrystalline silicon films. By tailoring the applied voltage waveforms, the authors demonstrated that the ion bombardment energy at the substrate can be modified, thus allowing control over the mode of silicon film grown. With the practical importance of these non-

\footnotetext{
${ }^{\text {a) }}$ Author to whom correspondence should be addressed. Electronic mail: trevor.lafleur@lpp.polytechnique.fr.
}

sinusoidal waveforms, and the demonstration of their production experimentally, ${ }^{4,9}$ it is of immense interest to investigate these systems in detail since by changing the excitation voltage waveform, it now potentially becomes possible to control the rate of sheath expansion and contraction on a level not possible in conventional CCP systems. In this letter, we investigate the use of pulse-type non-sinusoidal voltage waveforms using particle-in-cell (PIC) simulations, ${ }^{10}$ and directly investigate the effect this has on the plasma discharge, focussing explicitly on how the power deposition and electrical properties are modified.

We make use of a modified version of the custom developed PHOENIX $1 \mathrm{D}$ code, ${ }^{11}$ and simulate a $1 \mathrm{D}$ plasma reactor together with an external circuit containing a bias capacitor, $C_{B}$ (with a value of $10 \mathrm{nF} \mathrm{m} \mathrm{m}^{-2}$ ). The right-hand boundary is grounded, while the left-hand boundary can float (due to the presence of the bias capacitor). A schematic of the reactor and circuit is shown in Fig. 1(a). An rf voltage source in the circuit is used to apply a sine, or Gaussian pulse waveform (at the same voltage amplitude); a number of which are shown in Fig. 1(b). The repetition frequency of the pulses is $13.56 \mathrm{MHz}$, while pulse widths, expressed as the full width half maximum $(\Delta \tau)$, between 5 and $20 \mathrm{~ns}$ are used. The form of each pulse is $V(t)=V_{0} \exp \left[-\alpha\left(t-t_{0}\right)^{2}\right]$, where $V_{0}$ is the voltage amplitude, $\alpha$ is a parameter controlling the width $\Delta \tau$, and $t_{0}$ is the time of the pulse maximum.

A Monte Carlo collision algorithm ${ }^{12}$ is used in the simulations, with elastic, excitation, and ionization electronneutral reactions, and charge-exchange and elastic ionneutral reactions. The neutral argon gas used is assumed to be uniformly distributed with a temperature of $300 \mathrm{~K}$. Electrons are reflected from the boundaries with a probability of 0.2 , while the secondary electron emission coefficient due to ion bombardment is 0.1. Emitted electrons are chosen from a half-Maxwellian distribution with a temperature of $2 \mathrm{eV}$. We use about $1 \times 10^{5}$ particles, with time steps between $1 \times 10^{-11} \mathrm{~s}$ and $4 \times 10^{-11} \mathrm{~s}$, and about 400-800 grid points. The distance between the electrodes is $2 \mathrm{~cm}$ (and some limited test cases with $6.7 \mathrm{~cm}$ ). Due to the presence of the bias 


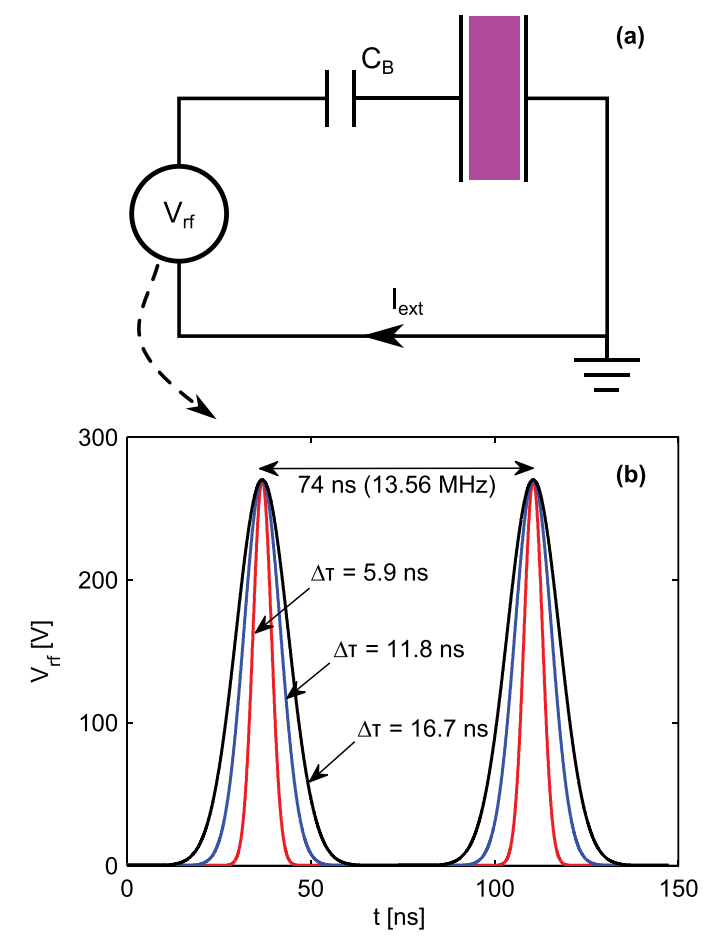

FIG. 1. (a) Schematic of the PIC model and external circuit, showing the rf voltage source $\left(V_{r f}\right)$, and bias capacitor $\left(C_{B}\right) . I_{e x t}$ is the current flowing in the external circuit. (b) Examples of Gaussian excitation voltage waveforms with different full width half maxima $(\Delta \tau)$ supplied by the rf voltage source. The repetition frequency is $13.56 \mathrm{MHz}$.

capacitor, the voltage on the powered electrode is not known a priori, but is solved using the method in Ref. 13. Here, the voltage is obtained directly by decomposing the field solver into a solution of the Poisson equation (with zero boundary conditions), and the Laplace equation (with the external circuit and other boundary conditions). Because of the presence of the bias capacitor, and the changing capacitive voltage divider due to the plasma sheaths, the powered electrode voltage $\left(V_{e}\right)$ is less than the full voltage amplitude $\left(V_{0}\right)$.

Although pressures of between 20 and 1000 mTorr were used, we choose to focus mainly on the results at $100 \mathrm{mTorr}$. Initially, secondary electron emission and electron reflection is turned off. Figure 2 shows simulation results for a sine wave excitation, and also for a positive pulse excitation. The voltage amplitude is $V_{0}=270 \mathrm{~V}$, and $\Delta \tau=11.8 \mathrm{~ns}$ for the pulse case. The sine case has no dc bias, as expected from the geometrical symmetry of the system, and thus the timeaveraged sheath potential differences are equal. The pulse excitation however develops a significant dc bias of about $-120 \mathrm{~V}$; characteristic of the EAE effect. The time-averaged plasma potential is also asymmetric, and the potential drop across the sheath at the powered electrode is much higher than that at the ground electrode. The density is also skewed, with a wider sheath at the powered electrode. For both voltage excitations, the maximum densities are similar, with a value of about $7-9 \times 10^{15} \mathrm{~m}^{-3}$. The results for the sine case are very close to those from other PIC simulations under similar operating conditions. ${ }^{14}$

The formation of a negative bias for the positive pulse case in Fig. 2 is consistent with recent experimental measurements (using a similar waveform) in Ref. 9. If a negative pulse is simulated instead, a mirror-image of the results in
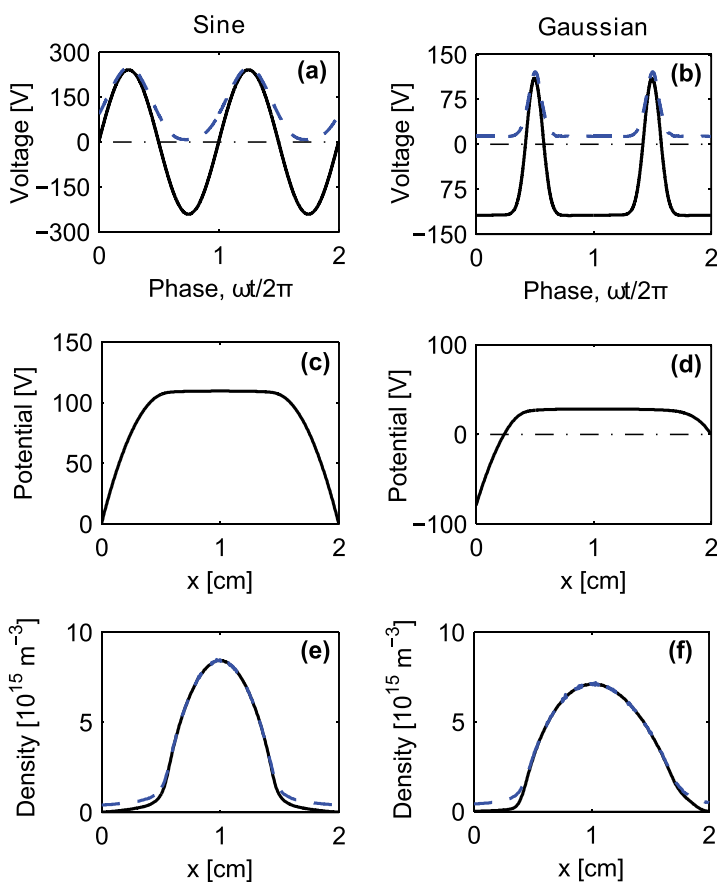

FIG. 2. Electrode voltage (solid line) and central plasma potential (dashed line) [(a) and (b)], time-averaged plasma potential profiles [(c) and (d)], and time-averaged plasma density profiles [(e) and (f); solid line for electron density, and dashed line for ion density] for a sinusoidal and a positive Gaussian voltage excitation. The pressure is $100 \mathrm{mT}$ orr and $V_{0}=270 \mathrm{~V}$.

Fig. 2 is obtained (this is also true for all other results presented below), and a positive dc bias now forms. Use of a negative pulse in Ref. 9 produced a very small dc bias close to zero, but the experimental system in that case is not geometrically symmetric.

Figure 3 shows the plasma and electrical response to varying the pulse width. The electrode voltage, together with the bias voltage, is observed to remain essentially constant at around $220 \mathrm{~V}$ and $-120 \mathrm{~V}$, respectively. The maximum plasma density increases rapidly as the pulse width is decreased, going from about $5 \times 10^{15} \mathrm{~m}^{-3}$ at $\Delta \tau=17 \mathrm{~ns}$ to $16 \times 10^{15} \mathrm{~m}^{-3}$ at $\Delta \tau=6 \mathrm{~ns}$. Similar behaviour is seen for the electron and ion power absorption, which are found from a temporal and spatial integration of $\mathbf{E} \cdot \mathbf{J}$, where $\mathbf{E}$ is the electric field and $\mathbf{J}$ is the electron (or ion) current density. These results are in stark contrast to the PIC simulations recently presented by Donkó. ${ }^{5,8}$ There, a voltage excitation of the form $V(t)=V_{0}[\cos (\omega t+\theta)+\cos 2 \omega t]$ was used, and as the phase shift $\theta$ was changed, the bias voltage could be controlled while keeping the plasma density and power deposition almost constant. If a similar voltage form is used in our simulations here, we observe a similar effect. It is thus clear that the pulse excitation produces very different behaviour. Since $\Delta \tau$ affects the rate of sheath expansion and collapse (in a manner not possible by changing $\theta$ in the above waveform), we now investigate the power deposition profiles in more detail.

Figure 3(d) shows the time-averaged electron power deposition for a number of pulse widths. As seen, the heating is localised to the sheath regions, and increases substantially as the pulse width decreases. We can demonstrate that this increased deposition is due to enhanced collisionless sheath heating, by observing the time-varying electron distribution function. This is shown in Figure 4(a), where the negative 

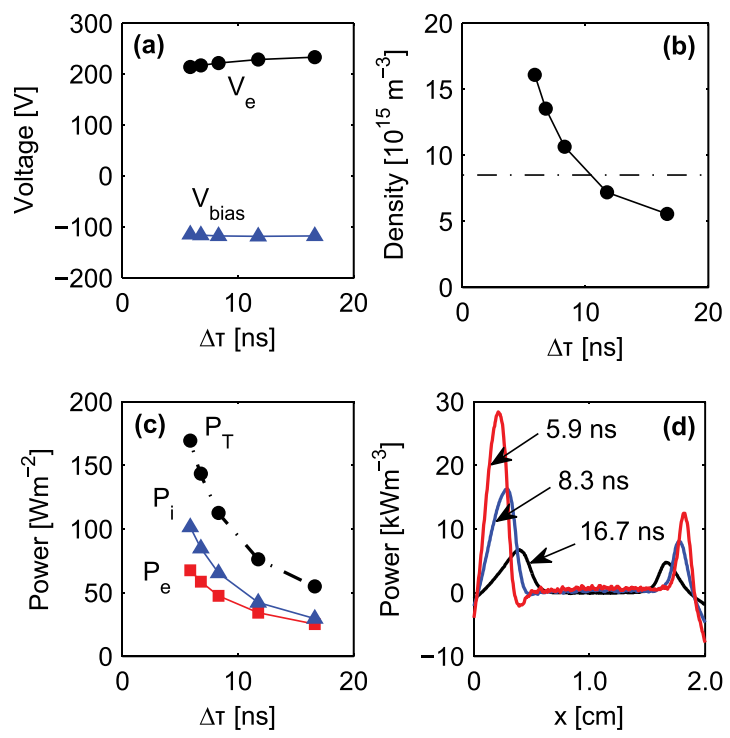

FIG. 3. (a) Electrode $\left(V_{e}\right)$ and bias voltage $\left(V_{\text {bias }}\right)$, (b) maximum plasma density, and (c) electron power absorption $\left(P_{e}\right)$, ion power absorption $\left(P_{i}\right)$, and total power absorption $\left(P_{T}=P_{e}+P_{i}\right)$ as a function of pulse width $(\Delta \tau)$. The solid lines in (a)-(c) serve as a visualization aid, while the dotteddashed line in (b) shows the maximum plasma density for the sinusoidal voltage excitation. The dotted-dashed line in (c) is the total power absorption found from a time integration of the instantaneous power $P(t)=V_{e}(t) I_{e x t}(t)$. (d) Spatial (time-averaged) electron power absorption for three different pulse widths. The pressure is $100 \mathrm{mTorr}$, and $V_{0}=270 \mathrm{~V}$.

energies are for electrons moving to the left and the positive energies are for electrons moving to the right (i.e., away from the sheath at the powered electrode). The distribution functions in Fig. 4(a) are shown at 3 locations in the pulse: (A) rising edge of the pulse (when the sheath is collapsing), (B) maximum value of the pulse (when the sheath has fully collapsed), and (C) lowering edge of the pulse (as the sheath is expanding). As seen, a significant perturbation to the distribution function occurs at high energies as the sheath is expanding; behaviour that is typically associated with collisionless sheath heating. Figure 4(b) shows the right moving electrons for a number of pulse widths, where it is seen that the temperature of the hot tail $\left(T_{e_{H}}\right)$ of the distribution function increases as the pulse width decreases, further signalling an enhanced sheath heating process.

By turning secondary electron emission and electron reflection on, similar results to those presented above were obtained, except that the power deposition was seen to increase by about $10 \%-30 \%$, similar to that in standard sinusoidal CCP discharges at this frequency and pressure. ${ }^{1}$ Simulations were also run with a longer system length of $6.7 \mathrm{~cm}$ and a pressure of $20 \mathrm{mTorr}$, and similar results were obtained. Finally, a number of additional simulations using symmetric triangular pulses instead of Gaussian pulses were performed, and again similar results were observed; as the pulse width is decreased, the power deposition is enhanced.

The above results consistently demonstrate that reducing the pulse width allows for a larger plasma density and increased power deposition due to enhanced sheath heating. All of this occurs for essentially the same applied voltage amplitude, bias voltage, and pressure, and is thus potentially of great practical importance, since it presents a possible solution to help overcome the power deposition limits of
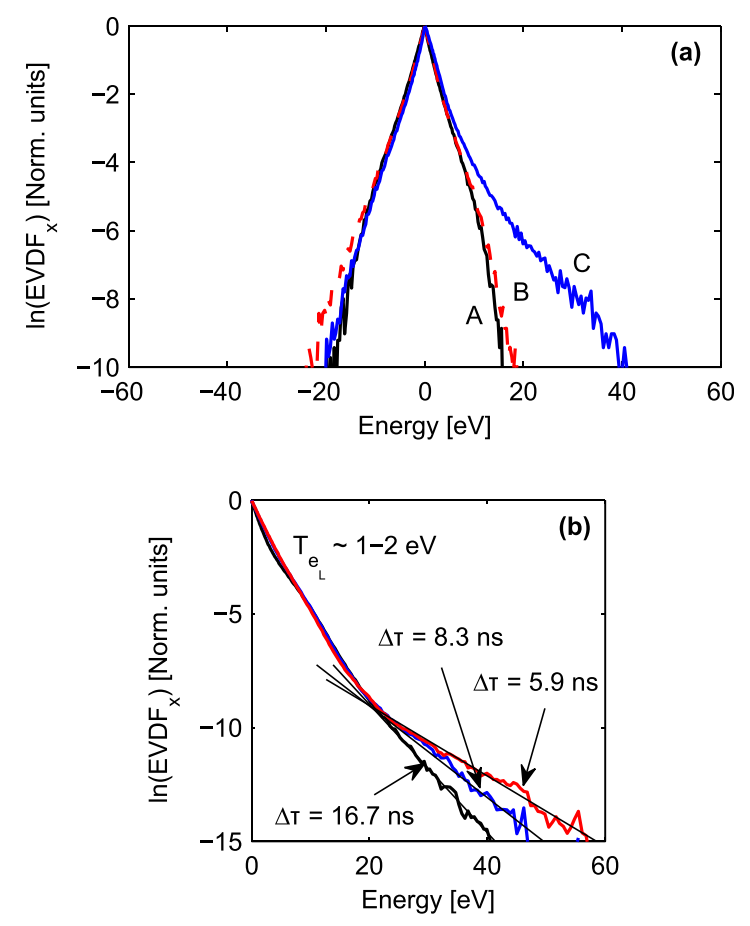

FIG. 4. (a) Electron velocity distribution function in the x-direction at three rf phase locations: (A) rising edge of the Gaussian pulse, (B) maximum of the pulse, and (C) lowering edge of the pulse. Negative energies represent electrons moving to the left, while positive energies represent electrons moving to the right. The pressure is $100 \mathrm{mTorr}, V_{0}=270 \mathrm{~V}$, and the pulse width is $\Delta \tau=8.3 \mathrm{~ns}$. (b) Time-averaged electron velocity distribution functions for electrons moving to the right for three pulse widths. The temperature of the high-energy electron tail regions $\left(T_{e_{H}}\right.$; indicated by the straight lines) for pulse widths of $\Delta \tau=16.7 \mathrm{~ns}, \Delta \tau=8.3 \mathrm{~ns}$, and $\Delta \tau=5.9 \mathrm{~ns}$ is $3.6 \mathrm{eV}, 4.9 \mathrm{eV}$, and $7.3 \mathrm{eV}$, respectively.

current CCP systems. Although enhanced power deposition has been theoretically predicted in non-harmonic dual frequency CCP systems, ${ }^{15}$ what remains now is for an experimental campaign to validate the above simulation results for pulse-type excitations. Significant practical challenges in this regard, however, remain with the impedance matching of the non-sinusoidal voltage source and the plasma. ${ }^{9}$

Recent PIC simulations by Donkó ${ }^{16}$ have shown that the nonlinear plasma series resonance (NPSR) effect ${ }^{17}$ can be self-excited in electrically asymmetric discharges. This resonance was demonstrated by the presence of high frequency components in the electron heating rates. In the present work, we observe behaviour qualitatively similar; as the pressure is decreased, significant high frequency modulation in both the electron heating rates and discharge current develops. These facts suggest that the NPSR is likely present in the Gaussian-excited discharges. In the current investigation, however, we have not attempted to explore the exact nature of the stochastic sheath heating process, be it Fermi acceleration, pressure heating, NPSR, or enhancement of the former two by NPSR. ${ }^{18}$ Because of the continuing debate within the literature, more careful work is needed to determine the exact type of electron heating mechanism, and thus whether NPSR does indeed play a significant role.

The simulation results presented here only consider electrostatic effects. At higher frequencies and densities, it is well-known that electro-magnetic (EM) effects (standing wave and skin depth effects) become important, ${ }^{19}$ and that 
this can change the power deposition and plasma behaviour. $^{20}$ In Ref. 19, it was estimated that skin depth effects can be neglected if $c / \omega_{p} \gg 0.45 \sqrt{R d}$, where $c$ is the speed of light, $\omega_{p}$ is the electron plasma frequency, $R$ is the discharge radius, and $d$ is the plasma half-width (after subtracting the sheath width). Using a representative value of $R=25 \mathrm{~cm}$, and $d \sim 7.5 \mathrm{~mm}$ (from the simulations), we estimate that skin depth effects become important for densities above $3-6 \times 10^{16} \mathrm{~m}^{-3}$; values larger than that investigated here. What is not clear yet though is whether the additional inductive electric fields (and associated magnetic fields) from surface currents on the electrodes, can perturb the electron trajectories sufficiently to significantly affect the stochastic sheath heating process itself. Since EM effects are difficult to include in PIC simulations, this remains a challenge for future work.

In summary, we have shown that the power deposition in CCP discharges can be increased due to enhanced electronsheath heating, resulting from the use of Gaussian-shaped excitation voltages. This potentially allows a level of control over the power deposition, plasma density, and ion flux not possible in conventional sinusoidally excited CCP systems.

${ }^{1}$ M. A. Lieberman and A. J. Lichtenberg, Principles of Plasma Discharges and Materials Processing (Wiley, NJ, 2005).

${ }^{2}$ B. G. Heil, U. Czarnetzki, R. P. Brinkmann, and T. Mussenbrock, J. Phys. D: Appl. Phys. 41, 165202 (2008).
${ }^{3}$ B. G. Heil, J. Schulze, T. Mussenbrock, R. P. Brinkmann, and U. Czarnetzki, IEEE Trans. Plasma Sci. 36, 1404 (2008).

${ }^{4}$ J. Schulze, E. Schüngel, and U. Czarnetzki, J. Phys. D: Appl. Phys. 42, 092005 (2009).

${ }^{5}$ Z. Donkó, J. Schulze, B. G. Heil, and U. Czarnetzki, J. Phys. D: Appl. Phys. 42, 025205 (2009).

${ }^{6}$ Z. Donkó, J. Schulze, P. Hartmann, I. Korolev, U. Czarnetzki, and E. Schüngel, Appl. Phys. Lett. 97, 081501 (2010).

${ }^{7}$ J. Schulze, E. Schüngel, U. Czarnetzki, M. Gebhardt, R. P. Brinkmann, and T. Mussenbrock, Appl. Phys. Lett. 98, 031501 (2011).

${ }^{8}$ E. Schüngel, J. Schulze, Z. Donkó, and U. Czarnetzki, Phys. Plasmas 18, 013502 (2011).

${ }^{9}$ E. V. Johnson, T. Verbeke, J. C. Vanel, and J. P. Booth, J. Phys. D: Appl. Phys. 43, 412001 (2010).

${ }^{10}$ C. K. Birdsall and A. B. Langdon, Plasma Physics Via Computer Simulation (McGraw-Hill, New York, 1985).

${ }^{11}$ S. D. Baalrud, T. Lafleur, R. W. Boswell, and C. Charles, Phys. Plasmas 18, 063502 (2011).

${ }^{12}$ V. Vahedi and M. Surendra, Comput. Phys. Commun. 87, 179 (1995).

${ }^{13}$ V. Vahedi and G. DiPeso, J. Comput. Phys. 131, 149 (1997).

${ }^{14}$ V. Vahedi, G. DiPeso, C. K. Birdsall, M. A. Lieberman, and T. D. Rognlien, Plasma Sources Sci. Technol. 2, 261 (1993).

${ }^{15}$ M. M. Turner and P. Chabert, Phys. Rev. Lett. 96, 205001 (2006).

${ }^{16}$ Z. Donkó, J. Schulze, U. Czarnetzki, and D. Luggenhölscher, Appl. Phys. Lett. 94, 131501 (2009).

${ }^{17}$ T. Mussenbrock and R. P. Brinkmann, Appl. Phys. Lett. 88, 151503 (2006).

${ }^{18}$ M. A. Lieberman, A. J. Lichtenberg, E. Kawamura, T. Mussenbrock, and R. P. Brinkmann, Phys. Plasmas 15, 063505 (2008).

${ }^{19}$ M. A. Lieberman, J. P. Booth, P. Chabert, J. M. Rax, and M. M. Turner, Plasma Sources Sci. Technol. 11, 283 (2002).

${ }^{20}$ Y. Yang and M. J. Kushner, Plasma Sources Sci. Technol. 19, 055011 (2010). 